\title{
Chemometric Screening of Fourteen Essential Oils for Their Composition and Biological Properties
}

\author{
Filomena Monica Vella ${ }^{1} @$, Roberto Calandrelli ${ }^{1}{ }^{\infty}$, Domenico Cautela ${ }^{2}\left(\mathbb{D}\right.$, Immacolata Fiume $^{3}$, \\ Gabriella Pocsfalvi ${ }^{3, *(\mathbb{D})}$ and Bruna Laratta ${ }^{1, * \mathbb{D}}$ \\ 1 National Research Council (CNR), Institute of Research on Terrestrial Ecosystems (IRET), via P. Castellino, \\ 111-80131 Naples, Italy; filomenamonica.vella@cnr.it (F.M.V.); roberto.calandrelli@cnr.it (R.C.) \\ 2 Experimental Station for the Industry of the Essential Oils and Citrus Products (SSEA)-Special Agency of the \\ Chamber of Commerce of Reggio Calabria, via T. Campanella, 12-89125 Reggio Calabria, Italy; \\ dcautela@ssea.it \\ 3 National Research Council (CNR), Institute of Biosciences and BioResources (IBBR), via P. Castellino, \\ 111-80131 Naples, Italy; immacolata.fiume@ibbr.cnr.it \\ * Correspondence: gabriella.pocsfalvi@ibbr.cnr.it (G.P.); bruna.laratta@cnr.it (B.L.); \\ Tel.: +39-613-2585 (G.P.); +39-08-1613-2329 (B.L.)
}

Academic Editor: Yannis Dotsikas

Received: 6 October 2020; Accepted: 29 October 2020; Published: 4 November 2020

\begin{abstract}
Essential oils (EOs) obtained from aromatic plants are widely used worldwide, especially in cosmetic and food products due to their aroma and biological properties and health benefits. Some EOs have significant antimicrobial and antioxidant activities, and thus could effectively increase the shelf lives of foodstuff and beverages. In this study, fourteen essential oils (clove, eucalyptus, fennel, lavender, oregano, palmarosa, pepper, star anise, tea tree, turmeric, Chinese yin yang, Japanese yin yang, and ylang ylang) from different medicinal plant families were screened by gas-chromatography-mass spectrometry (GC-MS) for their different chemical profiles and bioassays were performed to assess their antifungal and antioxidant activities. The results obtained were assessed by principal component analysis (PCA). PCA distinguished six groups characterized by different terpene chemotypes. Amongst the EOs studied, the clove EO showed the strongest antioxidant activity characterized by an $\mathrm{EC}_{50}$ of $0.36 \mu \mathrm{L} / \mathrm{mL}$. The oregano $\mathrm{EO}$ had the greatest antiyeast activity characterized by a minimal inhibitory concentration of $10 \mu \mathrm{L} / \mathrm{mL}$. In conclusion, clove and oregano EOs are strong antifungal and antioxidant agents, respectively, with great potential in the food industry to avoid spoilage and to increase shelf life.
\end{abstract}

Keywords: essential oils; PCA analysis; GC-MS chemotyping; antioxidant activity; antiyeast activity

\section{Introduction}

Aromatic plants (APs) have been used since antiquity in folk medicine and as food preservatives due to their many biologically active components including phenolic compounds (e.g., phenolic acids, flavonoids, coumarins, lignans, stilbenes, and tannins), nitrogen-containing compounds (alkaloids, amines, and betalains) and terpenoids (including carotenoids). Essential oils (EOs) are the volatile fractions extracted from aromatic plants. EOs are highly complex natural mixtures of low-molecular mass volatile compounds such as terpenes and aldehydes that are responsible for the typical aroma of any APs [1-6]. EOs are produced by the different parts of plants, including buds, flowers, leaves, stems, twigs, seeds, fruits, roots, wood and bark, and stored in secretory cells, cavities, canals, epidermic cells and glandular trichomes [1,2,4-6]. Nearby 3000 EOs originated from 2000 plant species and are distributed around 60 botanical genera which are produced worldwide. In nature, EOs play important biological and ecological roles since they are directly involved in plant 
communication and thus are considered as the language of chemical signaling [7]. In plant defense, they act as antibacterial, antiviral, antifungal and insecticide agents. Additionally, some EOs may attract certain insects to promote the dispersion of pollens and seeds, or to repel undesirable others [3,7]. The term essential oil was used for the first time in the 16th century by Paracelsus von Hohenheim, who referred to the effective component of a drug as "Quinta essential" [8]. EOs have widely been used for centuries for their well-known virucidal, antibacterial, antifungal, anticancer, antioxidant, and antidiabetic activities in medicinal and pharmaceutical formulations, in perfumery, fragrance, and cosmetic industries, in aromatherapy and as food additives [1-4,6,9].

Traditionally the use of EOs is always narrow and limited to aromatherapy (baths, massages and wraps) and cosmetic applications (body creams, lotions and masks). Recently they have received considerable interest as new functional food ingredients. In fact, EOs are increasingly applied in foods and drinks which is boosted by a rising interest of consumers in natural supplements and the growing concern about potentially harmful synthetic additives [3,6,7]. Moreover, today many of the EOs are classified as Generally Recognized as Safe (GRAS) substances which makes them invaluable for use in food preparation, drugs, and cosmetics [2,10-12]. Many APs find their ways as resources of high-end value drugs and lead compounds too. Today there are a few hundred of EOs that are commercially important $[1,3,5,7]$; many are used in the medicinal and cosmetic fields, and in the food packaging industry.

Fresh and stored food, such as raw olive, dairy products (cheeses and yogurts), fruits and products (jams, canned fruits, dried fruits, glacéd fruits, and fruit salads), bakery products, alcoholic (wine and beer) and non-alcoholic (juice and soft drinks) beverages can undergo microbial contamination $[13,14]$. Food spoilage by microorganisms leads to significant quality deterioration and results in in huge economic losses [2]. In order to ensure the quality and safety of food products and thus to protect consumer health, effective antimicrobial and antioxidant measures are necessary. Heat treatment, pasteurization, and antiseptic packaging are the most used methods to avoid microbiological deterioration of fruit and vegetable juices and purees and to exclude spoilage as long as the packaging remains intact [15]. Spoilage is often a consequence of yeast growth in food and beverages leading to turbidity, sedimentation, gassiness and off-flavor [16]. Saccharomyces cerevisiae is widely found in the industrial fermentation of foods and beverages containing mono- and disaccharides. Common cases of food spoilage by yeast are reported in fresh sea food, packed meat, fresh and frozen vegetables, wines and dairy products. Foods and drinks that cannot be pasteurized are usually treated with preservatives to prevent the growth of mold, yeast and fungi. Primarily used chemical preservatives are weak acids, such as sorbic and benzoic acid, and their salts or sulfites. Recent studies, however, raise concerns about the use of some chemical preservatives such as benzoic acid that can degrade to benzene, a carcinogen substance when used in foods $[15,17]$. Further, to avoid fungal growth on crops, spraying fungicides over the fields is a frequently used practice. However, most of the fungicides as well as their degradation products can contaminate the foodstuff and thus their use is an issue for food safety.

In general, EOs display their antiyeast, antimold and antifungal activities by means of irreversible damages affecting the membrane permeability and osmotic balance of the cell. Furthermore, they were reported to be able to modify ion transport processes and interact with vital cellular constituents including membrane proteins, resulting in water imbalance, depletion of intracellular ATP concentration and finally causing cell death [2,18-20]. Furthermore, EOs possess antioxidant activity capable of counteracting the action of free radical-mediated lipid peroxidation with the resulting decrease in shelf stability of foodstuffs [21-24]. Most prominently, terpenes, terpenoids and phenylpropanoids, having lipophilic nature and showing remarkable antimicrobial activity, are the main active ingredient of EOs.

Here, we studied fourteen widely used EOs (clove, eucalyptus, fennel, lavender, oregano, palmarosa, pepper, star anise, tea tree, turmeric, Chinese yin yang, Japanese yin yang, and ylang ylang) extracted from five different medicinal plant families: Apiaceae, Lamiaceae, Myrtaceae, Poaceae and Rutaceae. 
These medicinal plant families are of considerable interest because they are rich in phenylpropanoids [1,2]. Volatile phenylpropanoids have multifaceted effects, which include antimicrobial and antioxidant properties. Thus, antiyeast and antioxidant activities alongside with their chemical compositions of the above EOs were investigated in order to evaluate their potential applicability as natural preservative agents in food and cosmetic industries, as well as in the human health field.

\section{Results and Discussion}

\subsection{EOs Chemical Composition and PCA Analysis}

The chemical composition of the 14 EOs was determined by gas-chromatography-mass spectrometry (GC-MS) analysis. Table 1 shows the list of compounds in order of their elution time. A total of 177 compounds were detected, with a number of identified molecules ranging between 7 and 62 for the individual EO. The most abundant components belong to the monoterpenoids and sesquiterpenes. In the clove EO, we detected fewer components, while the pepper EO was found to be the richest one, with 62 compounds identified.

The tea tree EO was rich in terpinen-4-ol (47.5\%) and $\gamma$-terpinene (17.2\%), in accordance with data reported by Noumi et al. [25] and Zhang et al. [26]. Star anise and fennel EOs had anethole as their main component, with values of $75.0 \%$ and $50.8 \%$, respectively $[4,27,28]$. D-limonene was particularly abundant in the terpene profile of the bitter orange EO with a percentage of $93.9 \%[29,30]$. Moreover, the pepper EO extracted in our laboratory showed a percentage of D-limonene of $21.3 \%$ similar to o-cymene, which represented 23.9\% [31,32]. EOs eucalyptus and Chinese yin yang had eucalyptol as the most abundant compound $-88.3 \%$ and $34.5 \%$, respectively $[23,25]$. For the mixed Chinese yin yang, the results showed that is mainly consisted of EO from Eucalyptus globulus. The terpene profiles of the turmeric, clove, oregano and palmarosa EOs were found to be similar to those present in the literature data. In particular, turmeric was mainly composed by $29.3 \%$ of ar-tumerone and $28.8 \%$ of $\alpha$-turmerone [23], and clove was made up of eugenol with a percentage of $82.7 \%[6,22,33,34]$. Furthermore, the oregano EO had $69.6 \%$ carvacrol $[6,33]$ and palmarosa EO had $82.6 \%$ geraniol [35]. The Japanese yin yang EO contained menthol (35.6\%) and L-menthone (33.8\%), characteristic molecules of the Mentha arvensis plant from which the EO was already characterized [36,37]. Ylang ylang EO showed $\beta$-copene (19.5\%) and caryophyllene (15.5\%) and this chemotype was different from that reported in other studies in the literature [23]. The differences in terpene composition could be due to the intrinsic characteristics of plant such as age, stage of development, degree of maturity, and genetic variability, from which the EO used in the present study was extracted. Moreover, it is well-documented that environmental conditions (climate, soil, altitude, latitude, etc.) and the method used for the extraction affect the chemical composition of EOs [1,7]. Finally, the lavender EO was mainly composed of the monoterpene linalool (23.3\%) and of the $p$-anisaldehyde at 30.3\% which was for the first time reported in lavender EO, unlike what has been described in the literature so far [38-40].

We found that the GC-MS profile was characteristic to each EO. The chemical profile obtained by GC-MS analysis (Table 1) contains quantitative data of the different plant metabolites present in the EOs that enables their molecular-based classification, which can be explored by means of multivariate statistical techniques. Here principal component analysis (PCA), one of the most widely used techniques, was used to elaborate possible relationships between EOs and their terpenic composition [41,42]. PCA reduces the complexity of original data meanwhile retaining most of the information (variables) to emphasize variation and bring out strong patterns in a dataset. Recently PCA has been shown to be very useful when combined with targeted and untargeted analytical fingerprinting techniques and applied to herbal extracts and EOs [43]. 
Table 1. Chemical composition of 14 essential oils (Eos) expressed as relative concentrations ( $\%$ = peak area percent; RT = Retention Time).

\begin{tabular}{|c|c|c|c|c|c|c|c|c|c|c|c|c|c|c|c|}
\hline $\begin{array}{c}\mathrm{RT} \\
(\min )\end{array}$ & Compound & $\begin{array}{c}\text { Bitter } \\
\text { Orange } \\
(\%)\end{array}$ & $\begin{array}{c}\text { Clove } \\
(\%)\end{array}$ & $\begin{array}{c}\text { Eucalyptus } \\
(\%)\end{array}$ & $\begin{array}{c}\text { Fennel } \\
(\%)\end{array}$ & $\begin{array}{c}\text { Lavender } \\
(\%)\end{array}$ & $\begin{array}{c}\text { Oregano } \\
(\%)\end{array}$ & $\begin{array}{c}\text { Palmarosa } \\
(\%)\end{array}$ & $\begin{array}{c}\text { Pepper } \\
(\%)\end{array}$ & $\begin{array}{c}\text { Star } \\
\text { Anise } \\
(\%)\end{array}$ & $\begin{array}{l}\text { Tea Tree } \\
(\%)\end{array}$ & $\begin{array}{c}\text { Turmeric } \\
(\%)\end{array}$ & $\begin{array}{c}\text { Chinese } \\
\text { Yin Yang } \\
(\%)\end{array}$ & $\begin{array}{c}\text { Japanese } \\
\text { Yin Yang } \\
(\%)\end{array}$ & $\begin{array}{c}\text { Ylang } \\
\text { Ylang } \\
(\%)\end{array}$ \\
\hline 5.37 & $\beta$-Phellandrene & 0.206 & & & 0.212 & & & & 0.937 & & 0.079 & & & 0.323 & \\
\hline 5.53 & $\beta$-Pinene & 0.602 & & 0.183 & 0.825 & & & & 0.784 & & 0.141 & 0.159 & 2.929 & 11.953 & 0.074 \\
\hline 5.54 & Amyl vinyl carbinol & & & & & 0.270 & 0.399 & & & & & & & & \\
\hline 5.75 & Ethyl amyl ketone & & & & & 0.975 & 0.102 & & & & & & & & \\
\hline 5.86 & $\beta$-Myrcene & 0.971 & & 0.111 & 0.509 & & 1.567 & 0.383 & 2.904 & & 0.105 & & 0.857 & & 0.092 \\
\hline 6.03 & Amyl ethyl carbinol & & & & & 0.306 & & & & & & & & 0.127 & \\
\hline 6.30 & $(-)-\alpha$-thujene & & & & 2.462 & & 0.200 & & 3.072 & & 0.327 & 10.683 & 0.534 & & \\
\hline 6.51 & 3-Carene & & & & & 0.267 & & & & 0.210 & & 0.222 & 5.412 & & \\
\hline 6.77 & $\alpha$-Terpinolene & & & & & & & & & & 7.097 & & & & \\
\hline 6.86 & Menthene & & & & & & & & & & 0.055 & & & & 4.464 \\
\hline 6.91 & $(+)$-4-Carene & & & & & & 1.396 & & & & & & & & \\
\hline 7.02 & o-Cymene & & & 7.865 & 1.615 & 0.443 & 14.002 & & 23.928 & 0.340 & 11.591 & 2.744 & 2.806 & 0.084 & \\
\hline 7.17 & D-Limonene & 93.947 & & 0.904 & 9.004 & 0.228 & 0.358 & 0.159 & 21.281 & 1.152 & 1.992 & 0.738 & 8.937 & 3.127 & \\
\hline 7.28 & Eucalyptol & & & 89.338 & & 3.578 & 0.181 & & 0.157 & 0.302 & 2.201 & 2.609 & 34.462 & 0.099 & 0.301 \\
\hline 7.49 & 2-Norpinene & & & & & & & 0.169 & & & & & & & \\
\hline 7.68 & Lavender lactone & & & & & 0.400 & & & & & & & & & \\
\hline 7.87 & $\beta$-o-Cymene & & & & & & & 0.380 & & & & & & & \\
\hline 8.25 & $\gamma$-Terpinene & & & & 0.326 & & 7.504 & & & & 17.225 & & 1.264 & & \\
\hline 8.29 & $\alpha$-Pinene & & & & 0.225 & & & & & & & 0.331 & & & \\
\hline 8.81 & cis-Linaloloxide & & & & & 5.848 & & & & & & & & 0.058 & \\
\hline 9.30 & Fenchone & & & & 18.779 & & & & & & & & & & \\
\hline 9.32 & Isoterpinolene & & & & & & & & & & & 0.407 & 1.294 & & \\
\hline 9.35 & Linalool oxide & & & & & 5.203 & & & & & & & & & 1.695 \\
\hline 9.68 & Linalool & 0.243 & & 0.079 & & 23.294 & 1.351 & 2.548 & & 0.300 & & & 0.511 & 0.218 & 9.471 \\
\hline 9.75 & Perillen & & & & & & & & 0.072 & & & & & & \\
\hline 9.89 & $\delta$-Thujone & & & & & & & & & & & & 0.262 & & \\
\hline 10.19 & $\alpha$-Fenchol & & & & & 0.898 & & & & & 0.066 & & & & \\
\hline 10.4 & trans- $p$-Mentha-2,8-dienol & 0.243 & & & & & & & & & & & & & \\
\hline 10.52 & 3-Octanol-acetate & & & & & 0.138 & & & & & & & & & \\
\hline 10.8 & Limonene oxide, cis- & 0.559 & & & & & & & & & 0.207 & & & & \\
\hline 10.87 & $p$-Mentha-2,8-dien-1-ol & 0.370 & & & & & & & & & & & & & \\
\hline 10.94 & Limonene oxide, trans- & 0.517 & & & & & & & & & & & & & \\
\hline 11.00 & Isopinocarveol & & & 0.180 & & & & & & & 0.642 & & & & \\
\hline 11.14 & Camphor & & & & 0.364 & 4.031 & & & & & 0.057 & & 1.049 & & \\
\hline 11.18 & Thujol & & & & & 0.101 & & & & & 0.323 & & & 1.217 & \\
\hline 11.39 & Citronellal & & & & & & & & & & & & 7.528 & & \\
\hline 11.43 & L-Menthone & & & & & & & & & & & & 2.357 & 33.836 & \\
\hline 11.73 & $p$-Menthan-3-one & & & & & & & & & & & & 1.275 & & \\
\hline 11.77 & Menthol & & & & & & & & & & & & & 35.623 & \\
\hline 11.86 & endo-Borneol & & & & & 2.377 & 0.183 & & & & & & & & 1.755 \\
\hline 11.92 & Carvenone & & & & & 0.475 & & & 0.123 & & & & & & \\
\hline 12.10 & $p$-Menthan-1-ol & & & & & & & & & & & & 4.582 & & \\
\hline
\end{tabular}


Table 1. Cont.

\begin{tabular}{|c|c|c|c|c|c|c|c|c|c|c|c|c|c|c|c|}
\hline $\begin{array}{c}\mathrm{RT} \\
\text { (min) }\end{array}$ & Compound & $\begin{array}{c}\text { Bitter } \\
\text { Orange } \\
(\%)\end{array}$ & $\begin{array}{l}\text { Clove } \\
(\%)\end{array}$ & $\begin{array}{l}\text { Eucalyptus } \\
(\%)\end{array}$ & $\begin{array}{c}\text { Fennel } \\
(\%)\end{array}$ & $\begin{array}{l}\text { Lavender } \\
(\%)\end{array}$ & $\begin{array}{l}\text { Oregano } \\
(\%)\end{array}$ & $\begin{array}{l}\text { Palmarosa } \\
(\%)\end{array}$ & $\begin{array}{c}\text { Pepper } \\
(\%)\end{array}$ & $\begin{array}{c}\text { Star } \\
\text { Anise } \\
(\%)\end{array}$ & $\begin{array}{l}\text { Tea Tree } \\
\text { (\%) }\end{array}$ & $\begin{array}{c}\text { Turmeric } \\
(\%)\end{array}$ & $\begin{array}{c}\text { Chinese } \\
\text { Yin Yang } \\
(\%)\end{array}$ & $\begin{array}{c}\text { Japanese } \\
\text { Yin Yang } \\
(\%)\end{array}$ & $\begin{array}{c}\text { Ylang } \\
\text { Ylang } \\
(\%)\end{array}$ \\
\hline 12.13 & Neoisoisopulegol & & & & & & & & & & & & & 1.439 & \\
\hline 12.16 & Terpinen-4-ol & & & 0.279 & & 1.190 & 0.446 & & 0.087 & 0.138 & 47.525 & & 0.521 & & \\
\hline 12.34 & trans-3-Caren-2-ol & & & & & 0.079 & & & 0.054 & & & & & & \\
\hline 12.42 & Cryptone & & & & & 0.199 & & & 0.418 & & & & & & \\
\hline 12.57 & $\alpha$-Terpineol & & & & & & 0.071 & & & 0.178 & & & & & 0.120 \\
\hline 12.58 & Terpineol & 0.153 & & 0.868 & & 3.259 & & & & & 8.537 & & 1.745 & 7.064 & \\
\hline 12.8 & Estragole & & & & 14.115 & & & & & 0.256 & & & & & 0.147 \\
\hline 12.82 & Teresantalol & 0.125 & & & & & & & & & & & & & \\
\hline 12.85 & Verimol H & & & & & & & & & & 0.081 & & 0.079 & & \\
\hline 12.88 & Sabinol & 0.157 & & & & & & & 0.588 & & & & & & \\
\hline 13.13 & D-Verbenone & & & 0.060 & & 0.088 & & & & & & & & & \\
\hline 13.39 & trans-Carveol & 0.393 & & & & & & & & & & & & & \\
\hline 13.48 & Geranyl vinyl ether & & & & & 0.203 & & & & & & & & & \\
\hline 13.56 & Isoascaridol & & & & & & & & & & 0.055 & & & & \\
\hline 13.62 & Isobornyl formate & & & & & 0.544 & & & & & & & & & \\
\hline 13.63 & Nerol & 0.267 & & & & & & 0.362 & & & & & 1.627 & & \\
\hline 13.75 & Fenchyl acetate & & & & 0.114 & & & & & & & & & & \\
\hline 13.94 & Pulegone & & & & & 0.098 & & & 0.120 & & & & 0.097 & & \\
\hline 14.07 & (-)-Carvone & 0.320 & & 0.132 & & & 0.133 & & 0.075 & & & & & & \\
\hline 14.14 & Carvotanacetone & & & & & & & & 0.076 & & & & & & \\
\hline 14.29 & cis-Anethole & & & & 0.097 & & & & & 0.163 & & & & & \\
\hline 14.33 & Linalyl acetate & 0.484 & & & & & & & & & & & & & \\
\hline 14.36 & Piperitone & & & & & & & & & & & & & 0.208 & \\
\hline 14.37 & $p$-Anisaldehyde & & & & 0.466 & 30.273 & & & & 8.881 & & & & & \\
\hline 14.4 & Geraniol & & & & & & & 82.587 & & & & & 2.416 & 0.332 & 0.802 \\
\hline 14.48 & Benzaldehyde & & & & & & & & & 0.338 & & & & & \\
\hline 14.75 & $\alpha$-Citral & & & & & & & 0.825 & & & & & & & \\
\hline 14.77 & trans-Ascaridol glycol & & & & & 0.175 & & & & & 0.601 & & & & \\
\hline 14.88 & Phellandral & & & & & 0.470 & & & 0.179 & & & & & & \\
\hline 15.16 & Bornyl acetate & & & & & 0.222 & & & 0.142 & & & & 9.963 & & \\
\hline 15.25 & Anethole & 0.143 & & & 50.826 & & & & & 74.997 & 0.020 & & & & \\
\hline 15.26 & Lavandulyl acetate & & & & & 2.274 & & & & & & & & & \\
\hline 15.33 & Menthol acetate & & & & & & & & & & & & 0.836 & 3.503 & \\
\hline 15.57 & Linalyl formate & & & & & & & 0.145 & & & & & & & \\
\hline 15.76 & Carvacrol & & & & & & 69.641 & & 0.264 & & & & & & \\
\hline 15.95 & Carvomenthol & & & & & & & & 0.951 & & & & & & \\
\hline 16.25 & Hexyl tiglate & & & & & 0.207 & & & & & & & & & \\
\hline 16.43 & Elemene & & & & & & & & & & & & & & 0.090 \\
\hline 16.48 & Hotrienol & & & & & 2.476 & & & & & & & & & \\
\hline 16.54 & Limonene-diol & 0.163 & & & & & & & & & & & & & \\
\hline 16.71 & $\alpha$-Copaene & & & & & & & & 0.127 & & & & & & \\
\hline 16.72 & $\alpha$-Cubebene & & & & & & & & & & & & & & 0.119 \\
\hline
\end{tabular}


Table 1. Cont.

\begin{tabular}{|c|c|c|c|c|c|c|c|c|c|c|c|c|c|c|c|}
\hline $\begin{array}{c}\mathrm{RT} \\
(\min )\end{array}$ & Compound & $\begin{array}{c}\text { Bitter } \\
\text { Orange } \\
(\%)\end{array}$ & $\begin{array}{l}\text { Clove } \\
\text { (\%) }\end{array}$ & $\begin{array}{c}\text { Eucalyptus } \\
(\%)\end{array}$ & $\begin{array}{c}\text { Fennel } \\
(\%)\end{array}$ & $\begin{array}{l}\text { Lavender } \\
(\%)\end{array}$ & $\begin{array}{l}\text { Oregano } \\
\text { (\%) }\end{array}$ & $\begin{array}{l}\text { Palmarosa } \\
\quad(\%)\end{array}$ & $\begin{array}{c}\text { Pepper } \\
\text { (\%) }\end{array}$ & $\begin{array}{c}\text { Star } \\
\text { Anise } \\
(\%)\end{array}$ & $\begin{array}{l}\text { Tea Tree } \\
\text { (\%) }\end{array}$ & $\begin{array}{c}\text { Turmeric } \\
(\%)\end{array}$ & $\begin{array}{l}\text { Chinese } \\
\text { Yin Yang } \\
(\%)\end{array}$ & $\begin{array}{c}\text { Japanese } \\
\text { Yin Yang } \\
(\%)\end{array}$ & $\begin{array}{c}\text { Ylang } \\
\text { Ylang } \\
(\%)\end{array}$ \\
\hline 17.03 & Eugenol & & 82.723 & & & & & & & & & & 2.538 & & 0.348 \\
\hline 17.05 & Neryl acetate & & & & & 0.262 & & 0.088 & & & & & & & \\
\hline 17.25 & Ylangene & & & & & & & & & & & & & & 0.460 \\
\hline 17.31 & trans-Ascaridol glycol & & & & & 0.451 & & & & & & & & & \\
\hline 17.37 & Copaene & & 0.268 & & & & & & 0.306 & 6.792 & & & 0.144 & & 1.488 \\
\hline 17.49 & Geranyl acetate & & & & & 0.514 & & 10.122 & & & & & 0.159 & & 8.276 \\
\hline 17.56 & $(-)-\beta$-Bourbonene & & & & & 0.244 & & & & & & & & 0.092 & \\
\hline 17.57 & $p$-Acetonylanisole & & & & 0.061 & & & & & 5.613 & & & & & \\
\hline 17.69 & cis-Copaene & & & & & & & & & & & & & & 0.199 \\
\hline 17.73 & $\beta$-Elemene & & & & & & & & 0.647 & & & & 0.508 & & 0.501 \\
\hline 17.74 & Farnesol acetate & & & & & & & 0.058 & & & & & & & \\
\hline 17.82 & $\beta$-Copaene & & & & & & & & & & & & & & 0.060 \\
\hline 17.84 & Ascaridole epoxide & & & & & 0.340 & & & & & & & & & \\
\hline 18.07 & threo-Anethole glycol & & & & & & & & & 0.056 & & & & & \\
\hline 18.13 & Isoledene & & & & & & & & 1.206 & & & & & & \\
\hline 18.20 & Limonene oxide, cis- & & & & & 0.643 & & & & & & & & & \\
\hline 18.25 & trans- $\alpha$-Bergamotene & & & & & & & & & 0.055 & & & & & \\
\hline 18.37 & $\alpha$-Santalene & & & & & 0.444 & & & & & & & & & \\
\hline 18.40 & Caryophyllene & & 5.114 & & & & 2.103 & 1.789 & 2.823 & 0.060 & 0.723 & 0.370 & 1.482 & 0.294 & 15.541 \\
\hline 18.45 & $\alpha$-Bulnesene & & & & & & & & & & & & & & 0.084 \\
\hline 18.55 & 8-Hydroxy-carvotanacetone & & & & & 0.084 & & & & & & & & & \\
\hline 18.70 & cis- $\alpha$-Bergamotene & & & & & & & & & 0.168 & & & & & \\
\hline 18.83 & $\gamma$-Patchoulene & & & & & & & & & & & & 0.090 & & \\
\hline 18.94 & $( \pm)$-Thujpsadiene & & & & & & & & & & & 0.447 & & & \\
\hline 19.15 & Humulene & & 0.355 & & & & & 0.141 & 1.012 & & & & 0.214 & & 4.728 \\
\hline 19.29 & Alloaromadendrene & & & & & & & & 0.523 & & 0.090 & & & & \\
\hline 19.6 & Muurolene & & & & & & & & 0.616 & & & & & & 1.882 \\
\hline 19.71 & cis- $\beta$-Copaene & & & & & 0.201 & & & 0.643 & & & & 0.320 & & 19.461 \\
\hline 19.72 & $\alpha$-Curcumene & & & & & & & & & & & 2.745 & & & \\
\hline 19.73 & Mullilam diol & & & & & 0.164 & & & & & & & & & \\
\hline 19.99 & $\delta$-Curcumene & & & & & & & & & & & 2.281 & & & \\
\hline 20.00 & 7-epi-Sesquithujene & & & & & & & & & & & 0.218 & & & \\
\hline 20.02 & Germacrene D & & & & & & & & 0.822 & & & & & & 0.915 \\
\hline 20.05 & (+)-Ledene & & & & & & & & & & 0.080 & & & & \\
\hline 20.07 & $\gamma$-Elemene & & & & & & & & & & & & & & 0.622 \\
\hline 20.11 & $\alpha$-Muurolene & & & & & & & & 1.575 & & & & 0.121 & & 1.184 \\
\hline 20.21 & $\alpha$-Farnesene & & & & & & & & 0.407 & & & & & & 8.064 \\
\hline 20.27 & $\beta$-Bisabolene & & & & & 0.259 & 0.118 & & & & & 0.596 & & & \\
\hline 20.41 & $\gamma$-Muurolene & & & & & 0.378 & & & 1.628 & & & & & & \\
\hline 20.42 & Isogermacrene $\mathrm{D}$ & & & & & & & & & & & & & & 1.057 \\
\hline 20.43 & Cubenol & & & & & 0.513 & & & & & & & 0.120 & & \\
\hline 20.45 & 10-epi-Cubebol & & & & & & & & 0.271 & & & & & & \\
\hline
\end{tabular}


Table 1. Cont.

\begin{tabular}{|c|c|c|c|c|c|c|c|c|c|c|c|c|c|c|c|}
\hline $\begin{array}{c}\mathrm{RT} \\
(\min )\end{array}$ & Compound & $\begin{array}{c}\text { Bitter } \\
\text { Orange } \\
(\%)\end{array}$ & $\begin{array}{l}\text { Clove } \\
(\%)\end{array}$ & $\begin{array}{c}\text { Eucalyptus } \\
(\%)\end{array}$ & $\begin{array}{c}\text { Fennel } \\
(\%)\end{array}$ & $\begin{array}{l}\text { Lavender } \\
(\%)\end{array}$ & $\begin{array}{c}\text { Oregano } \\
(\%)\end{array}$ & $\begin{array}{l}\text { Palmarosa } \\
\quad(\%)\end{array}$ & $\begin{array}{l}\text { Pepper } \\
(\%)\end{array}$ & $\begin{array}{c}\text { Star } \\
\text { Anise } \\
(\%)\end{array}$ & $\begin{array}{l}\text { Tea Tree } \\
(\%)\end{array}$ & $\begin{array}{c}\text { Turmeric } \\
(\%)\end{array}$ & $\begin{array}{l}\text { Chinese } \\
\text { Yin Yang } \\
(\%)\end{array}$ & $\begin{array}{c}\text { Japanese } \\
\text { Yin Yang } \\
(\%)\end{array}$ & $\begin{array}{c}\text { Ylang } \\
\text { Ylang } \\
(\%)\end{array}$ \\
\hline 20.52 & Teresantalol & & & & & 0.201 & & & & & & & & & 4.016 \\
\hline 20.59 & $\delta$-Amorphene & & & & & & & & 3.855 & & & & 0.593 & & \\
\hline 20.60 & Sesquisabinene & & & & & & & & & & & 2.170 & & & \\
\hline 20.65 & Epizonarene & & & & & & & & & & & & & & 0.110 \\
\hline 20.66 & Eugenol acetate & & 10.281 & & & & & & & & & & & & \\
\hline 21.01 & $\alpha$-Calacorene & & & & & & & & 0.098 & & & & & & \\
\hline 21.13 & Elemyl acetate & & & & & & & & 2.673 & & & & 0.366 & & \\
\hline 21.54 & Palustrol & & & & & & & & 0.513 & & & & & & \\
\hline 21.67 & trans-pyran Linalool oxide & & & & & 0.868 & & & & & & & & & \\
\hline 21.69 & Germacrenol & & & & & & & & 1.300 & & & & & & \\
\hline 21.74 & (-)-Spathulenol & & & & & & & & 1.066 & & & & & & \\
\hline 21.88 & Caryophyllene oxide & & 1.197 & & & 3.148 & 0.224 & 0.244 & 1.195 & & & 0.372 & & & 0.193 \\
\hline 21.90 & (-)-Globulol & & & & & & & & & & 0.126 & & & & \\
\hline 22.01 & $( \pm)$-Dihydro-ar-turmerone & & & & & & & & & & & 0.444 & & & \\
\hline 22.03 & Epiglobulol & & & & & & & & 0.909 & & 0.053 & & & & \\
\hline 22.04 & Epi-Cadinol & & & & & & & & & & & & & & 0.422 \\
\hline 22.25 & Ledol & & & & & & & & 0.588 & & & & & & \\
\hline 22.32 & cis-Nuciferol & & & & & & & & & & & 0.708 & & & \\
\hline 22.40 & Humulene epoxide & & 0.061 & & & 0.272 & & & 0.473 & & & 0.159 & & & \\
\hline 22.57 & Junenol & & & & & & & & & & & & & & 0.300 \\
\hline 22.59 & $\alpha$-Corocalene & & & & & & & & 0.060 & & & & & & \\
\hline 22.72 & 10-epi-Cubenol & & & & & & & & & & & & & & 0.254 \\
\hline 22.79 & 10-epi- $\gamma$-Eudesmol & & & & & & & & 1.065 & & & & & & \\
\hline 22.91 & (Z)- $\gamma$-Atlantone & & & & & & & & 0.696 & & & 0.601 & & & \\
\hline 22.97 & $\tau$-Muurolol & & & & & & & & 1.243 & & & & & & 0.414 \\
\hline 22.98 & $\tau$-Cadinol & & & & & 0.063 & & & & & & & & & 0.994 \\
\hline 23.06 & Cubebene & & & & & & & & 0.349 & & & & & & \\
\hline 23.17 & 7-epi- $\beta$-Eudesmol & & & & & & & & 2.211 & & & & & & \\
\hline 23.22 & $\alpha$-Cadinol & & & & & & & & 2.944 & & & & & & 0.080 \\
\hline 23.28 & (Z)- $\alpha$-Atlantone & & & & & & & & 0.109 & & & 0.200 & & & \\
\hline 23.38 & $\alpha$-Turmerone & & & & & & & & & & & 28.801 & & & \\
\hline 23.46 & $a r$-Tumerone & & & & & & & & & & & 29.341 & & & \\
\hline 23.57 & $\begin{array}{l}\text { Isoaromadendrene } \\
\text { epoxide }\end{array}$ & & & & & 0.218 & & & & & & & & & \\
\hline 23.95 & 6-epi-Shyobunol & & & & & & & & 3.507 & & & & & & \\
\hline 24.07 & Curlone & & & & & & & & & & & 12.402 & & & \\
\hline 24.39 & trans-Farnesol & & & & & & & & & & & & & & 1.953 \\
\hline 24.77 & Oplopanone & & & & & & & & 0.330 & & & & & & \\
\hline 25.23 & Ascabiol & & & & & & & & & & & & & & 0.492 \\
\hline 25.32 & $\begin{array}{l}\text { trans-Z- } \alpha \text {-Bisabolene } \\
\text { epoxide }\end{array}$ & & & & & 0.064 & & & 1.034 & & & & & & \\
\hline 25.42 & $\begin{array}{l}\text { cis-Z- } \alpha \text {-Bisabolene } \\
\text { epoxide }\end{array}$ & & & & & 0.077 & & & & & & & & & \\
\hline
\end{tabular}


Table 1. Cont.

\begin{tabular}{|c|c|c|c|c|c|c|c|c|c|c|c|c|c|c|c|}
\hline $\begin{array}{c}\mathrm{RT} \\
\text { (min) }\end{array}$ & Compound & $\begin{array}{c}\text { Bitter } \\
\text { Orange } \\
(\%)\end{array}$ & $\begin{array}{l}\text { Clove } \\
(\%)\end{array}$ & $\begin{array}{l}\text { Eucalyptus } \\
(\%)\end{array}$ & $\begin{array}{c}\text { Fennel } \\
(\%)\end{array}$ & $\begin{array}{l}\text { Lavender } \\
(\%)\end{array}$ & $\begin{array}{l}\text { Oregano } \\
(\%)\end{array}$ & $\begin{array}{l}\text { Palmarosa } \\
(\%)\end{array}$ & $\begin{array}{l}\text { Pepper } \\
(\%)\end{array}$ & $\begin{array}{c}\text { Star } \\
\text { Anise } \\
(\%)\end{array}$ & $\begin{array}{c}\text { Tea Tree } \\
\text { (\%) }\end{array}$ & $\begin{array}{c}\text { Turmeric } \\
(\%)\end{array}$ & $\begin{array}{l}\text { Chinese } \\
\text { Yin Yang } \\
(\%)\end{array}$ & $\begin{array}{c}\text { Japanese } \\
\text { Yin Yang } \\
(\%)\end{array}$ & $\begin{array}{c}\text { Ylang } \\
\text { Ylang } \\
(\%)\end{array}$ \\
\hline 25.44 & Cubenol & & & & & & & & 1.126 & & & & & & \\
\hline 25.47 & (E)-Atlantone & & & & & & & & & & & 0.252 & & & \\
\hline 25.50 & (-)-Spathulenol & & & & & & & & 0.171 & & & & & & \\
\hline 26.11 & $\begin{array}{l}\text { Isoaromadendrene } \\
\text { epoxide }\end{array}$ & & & & & & & & 3.259 & & & & & & \\
\hline 26.28 & (+)-Spathulenol & & & & & & & & 0.191 & & & & & & 6.694 \\
\hline 27.12 & $\alpha$-Isonootkatol & & & & & & & & 0.062 & & & & & & \\
\hline 27.24 & Platambin & & & & & & & & 0.088 & & & & & & \\
\hline 27.56 & Ylangenol & & & & & & & & 0.064 & & & & & & \\
\hline
\end{tabular}


In this work, chemotyping of EOs was performed by studying the chemical variability between the samples and transforming the original correlated variables into new, reduced, artificial, and orthogonal ones, called principal components (F1 and F2 in Figure 1). The score-plot shows the relationship between different EOs (the observations) and the loading plot shows how strongly each characteristic influences a principal component (other variables). Graphically, EOs close to each other in the score-plots have similar chemotype. In this regard, the first two principal components (F1 and F2) had the highest share of the total variance $(33.6 \%)$, due to the relatively high number of variables analyzed (177 compounds). In fact, twelve principal components would explain $100 \%$ of the total variation. It is, however, worth noting that the number of principal components depends on the number of variables, as the percent of total variance increases with lower number of variables. Generally, by increasing the number of variables, the proportion of total variance decreases.

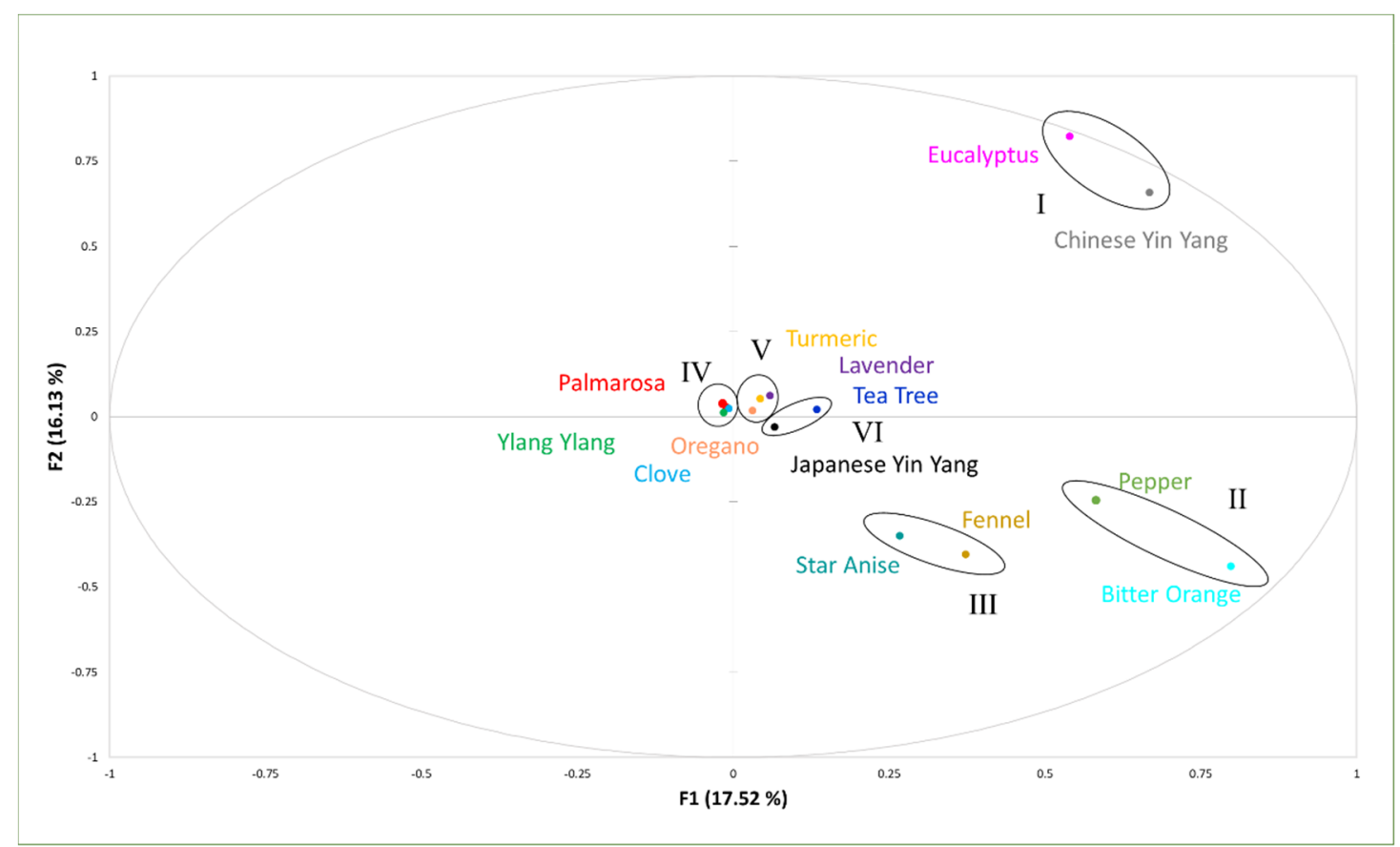

Figure 1. Principal component analysis (PCA) involving compositions of 14 EOs determined by gas-chromatography-mass spectrometry (GC-MS).

The first principal component F1, which accounted for $17.5 \%$ of the total variance, was positively correlated with eucalyptol (0.85), the major compound found in EOs of eucalyptus and Chinese yin yang, and with D-limonene (0.70), the main compound found in EOs of bitter orange and pepper. The F2, explaining $16.1 \%$ of the total variance, was positively correlated with carvacrol (0.9), the most abundant compound found in oregano EOs.

Based on the PCA analysis, the 14 EOs studied were grouped into six different clusters, which allowed to distinguish six chemotypes as shown in Figure 1. The first group (I) includes eucalyptus and Chinese yin yang, both characterized by a high content of eucalyptol, whereas the second one (II) was made up of bitter orange and pepper, which contains high percentage of D-limonene. Cluster III groups together star anise and fennel, based on the high quantity of anethole. Group IV was clustered by the presence of sesquiterpenes and was composed by cloves, palmarosa, and ylang ylang. Furthermore, it was observed that the EOs of turmeric, lavender, and oregano (Group V) were clustered because of the presence of eucalyptol and o-cymene. In the same way, the similarity between tea tree and Japanese yin yang can be observed (Group VI) due to the high relative percentage of the components eucalyptol and D-limonene. 


\subsection{Antiyeast Activity}

Problems with chemical preservatives and the growing demand of consumers for natural food additives have turned the attention to plant-derived natural antimicrobials such as EOs. In fact, EOs could represent an alternative to synthetic preservatives against spoilage due to yeasts and molds. The antiyeast effect of the 14 EOs was tested by the solid medium diffusion method. Table 2 shows the mean diameters of inhibition halos of each EO obtained on the food-spoiling yeast $\mathrm{S}$. cerevisiae. From the results obtained, yeast was resistant to star anise, turmeric, and ylang ylang, as the inhibition halo was completely absent. On the other hand, tea tree, fennel, lavender, pepper and Chinese yin yang showed inhibition halos ranging from 4 to $9 \mathrm{~mm}$. Oregano was the best performing inhibitor of S. cerevisiae growth among all the EOs analyzed by displaying a 35-mm inhibition halo (Table 2).

Table 2. Results of antiyeast activity of 14 essential oils using the solid medium diffusion assay.

\begin{tabular}{cc}
\hline Essential Oil & Mean Diameter $(\mathbf{m m})$ \\
\hline Bitter orange & 15 \\
Clove & 17 \\
Eucalyptus & 15 \\
Fennel & 5 \\
Lavender & 7 \\
Oregano & 35 \\
Palmarosa & 15 \\
Pepper & 4 \\
Star anise & N.D. \\
Tea tree & 8 \\
Turmeric & N.D. \\
Chinese yin yang & 9 \\
Japanese yin yang & 13 \\
Ylang ylang & N.D. \\
\hline
\end{tabular}

N.D. = inhibition zone diameter not detected.

The solid medium diffusion technique was proven to be a useful screening method in order to obtain preliminary data about the antiyeast effect of the EOs. The bitter orange, clove, eucalyptus, oregano, palmarosa, and Japanese yin yang EOs showed growth inhibition halos with diameters equal to or greater than $10 \mathrm{~mm}$. Their minimum inhibitory concentration (MIC) values (Table 3) were showed a more than $90 \%$ reduction in the measured absorbance [44].

Table 3. Minimum inhibitory concentration values (in $\mu \mathrm{L} / \mathrm{mL}$ ) of bitter orange, clove, eucalyptus, oregano, palmarosa and Japanese yin yang EOs.

\begin{tabular}{cc}
\hline Essential Oil & MIC $(\mu \mathrm{L} / \mathrm{mL})$ \\
\hline Bitter orange & 60 \\
Clove & 40 \\
Eucalyptus & 60 \\
Oregano & 10 \\
Palmarosa & 40 \\
Japanese yin yang & 80 \\
\hline
\end{tabular}

Amongst the 14 EOs tested, oregano showed the highest antiyeast activity, followed by clove, palmarosa, bitter orange, eucalyptus and Japanese yin yang. Thus, the oregano and clove EOs could be considered as potential antimicrobial agents to be used in the food bioconservation industry.

Oregano was the most active EO against the growth of S. cerevisiae, displaying an MIC of $10 \mu \mathrm{L} / \mathrm{mL}$ (Table 3); this result was in accordance with previous data [6,44]. Antiyeast activity of oregano EO could be associated with the high relative amount of carvacrol, as evidenced by its terpene 
composition (Table 1). In fact, carvacrol could be absorbed by the double phospholipidic layer of yeasts and could increase the fluidity and permeability of the membrane. Yeast cells, in the presence of carvacrol, have been shown to change the composition of their membrane's fatty acids as an adaptation mechanism to maintain the correct structure and function of the membrane $[6,45]$.

The antiyeast activity of clove and palmarosa EOs $(\mathrm{MIC}=40 \mu \mathrm{L} / \mathrm{mL})$ was owed to their most abundant components, eugenol and geraniol, respectively [6,45-49].

It is worth noting that the monoterpenes D-limonene, eucalyptol and menthol might be responsible for the antiyeast activity in bitter orange (MIC $=60 \mu \mathrm{L} / \mathrm{mL})$, eucalyptus (MIC $=60 \mu \mathrm{L} / \mathrm{mL}$ ) and Japanese yin yang $(\mathrm{MIC}=80 \mu \mathrm{L} / \mathrm{mL})$ EOs, respectively, as reported by Di Pasqua et al. [45] and Bassolé et al. [50].

\subsection{Antioxidant Activity}

Oxidation is one of the major causes of food degradation, which can occur along the entire food chain. In particular, oxidation is a process that causes unwanted quality changes, organoleptic variation, as well as affect the safety and nutritional value of foodstuffs. It occurs mainly through discoloration, odor generation and off-flavor, or through the formation of potentially toxic substances [2,12]. For this reason, the protection of food from oxidative deterioration is an important goal in food technology. EOs are regarded as GRAS thank to their chemical composition, biological effects and toxicity and are extensively exploited as natural antioxidants to be used in the food sector in contrast to chemical preservatives with known negative effects on human health [2,12].

$\mathrm{DPPH}$ is a stable free radical widely used to test the free-radical scavenging ability of various EOs. Clove, fennel, lavender, oregano, palmarosa, pepper, star anise, tea tree, turmeric, Chinese yin yang, and ylang ylang EOs were able to inhibit 50\% of the radical scavenging activity of DPPH, as can be seen in Table 4. On the contrary, bitter orange, eucalyptus, and Japanese yin yang revealed no antioxidant activity.

Table 4. Antioxidant activity of EOs measured by 2,2-diphenyl-1-picrylhydrazyl (DPPH) method and expressed as $\mathrm{EC}_{50}$.

\begin{tabular}{ccc}
\hline Essential Oil & EC $_{\mathbf{5 0}}(\mu \mathrm{L} / \mathrm{mL})$ & AAI \\
\hline Bitter orange & N.D. & N.D. \\
Clove & $0.36 \pm 0.02$ & 59.17 \\
Eucalyptus & N.D. & N.D. \\
Fennel & $90.86 \pm 0.14$ & 0.23 \\
Lavender & $665.54 \pm 0.50$ & 0.03 \\
Oregano & $11.58 \pm 0.22$ & 1.84 \\
Palmarosa & $950.52 \pm 0.71$ & 0.02 \\
Pepper & $62.10 \pm 0.23$ & 0.34 \\
Star anise & $500.57 \pm 0.33$ & 0.04 \\
Tea tree & $54.81 \pm 0.24$ & 0.39 \\
Turmeric & $24.99 \pm 0.44$ & 0.85 \\
Chinese yin yang & $5.35 \pm 0.13$ & 3.98 \\
Japanese yin yang & N.D. & N.D. \\
Ylang ylang & $12.71 \pm 0.17$ & 1.68 \\
\hline
\end{tabular}

AAI = antioxidant activity index; N.D. = antioxidant activity not detected. Values presented as mean \pm standard deviation.

The lowest $\mathrm{EC}_{50}$ values were found in clove $(0.36 \pm 0.02 \mu \mathrm{L} / \mathrm{mL})$ and Chinese yin yang $(5.35 \pm 0.13 \mu \mathrm{L} / \mathrm{mL})$, thus they are classified as very strong antioxidants, accordingly to Scherer and Godoy [51] and Cautela et al. [52].

Clove EO was renowned as one of the strongest antioxidants as previously reported by Teixera et al. [22], Misharina and Samusenko [24], Jirovetz et al. [34], and Wei and Shibamoto [53]. The high antioxidant activity of clove could be linked to the presence of eugenol as the EO's main constituent, revealed by the GC-MS analysis reported in Table 1. This compound is a phenylpropanoid 
derived from guaiacol and is known to possess antioxidant activity $[34,53,54]$. Clove EO, due to its high antioxidant activity, could be used as an antioxidant agent in order to prevent natural oxidation and deterioration of food and thus for increasing the shelf life.

Chinese yin yang was a mix of different EOs (Eucalyptus globulus, Cymbopogon citratus, Caryophylli aetheroleum, Mentha piperita, Pinus sylvestris, Salvia rosmarinus, Lavandula officinalis, Foeniculum vulgare, Salvia officinalis, Illicium verum, Mentha arvensis, and Abies siberica) and the very strong activity could be due to the synergistic and additive effects due to the combination of different EOs [15,55,56].

Additionally, oregano and ylang ylang exhibited a strong antioxidant activity and their $\mathrm{EC}_{50}$ values were $11.58 \pm 0.22$ and $12.71 \pm 0.17 \mu \mathrm{L} / \mathrm{mL}$, respectively. Oregano EO was known to possess antioxidant activity due to the presence of carvacrol, as reported in the literature [54], and our study was in accordance with this as this monoterpene phenol resulted to be very abundant (about 70\%), as depicted in Table 1. Ylang ylang EO was characterized as having caryophyllene as its main component and so the strong antioxidant activity of EO could be related to presence of this sesquiterpenes, as previously described [57].

Only turmeric displayed a moderate antioxidant activity with $24.99 \pm 0.44 \mu \mathrm{L} / \mathrm{mL}$, while the remaining EOs (fennel, lavender, palmarosa, pepper, star anise, and tea tree) revealed poor antioxidant activity, as reported in Table 4 . Overall, clove exhibited the highest antioxidant activity amongst the EOs studied, followed by Chinese yin yang, oregano, and ylang ylang.

\section{Materials and Methods}

\subsection{Chemicals}

2,2-Diphenyl-1-picrylhydrazyl (DPPH) and the MS-grade solvents were purchased from Sigma Chemical Co. (St. Louis, MO, USA). Malt extract, peptone, agar, DMSO and methanol were obtained from Carlo Erba Reagents (Milan, Italy).

\subsection{Essential Oils}

EOs of 13 plants were purchased from different companies, as reported in Table 5.

Table 5. Botanical, geographical, and commercial sources of EOs.

\begin{tabular}{|c|c|c|c|c|}
\hline Essential Oil & Species & Part of Plant & Country & Company \\
\hline Bitter orange & Citrus aurantium & Peels & Ivory Coast & Essenthya \\
\hline Clove & Eugenia caryophyllata & Buds & Sri Lanka & Primavera \\
\hline Eucalyptus & Eucalyptus globulus & Leaves and twigs & Hungary & $\begin{array}{l}\text { Phoenix } \\
\text { Pharma }\end{array}$ \\
\hline Fennel & Foeniculum vulgare & Seeds & Italy & Primavera \\
\hline Lavender & Lavandula officinalis & Flowers & Bulgaria & Primavera \\
\hline Oregano & Origanum vulgare & Flowering plants & Spain & Primavera \\
\hline Palmarosa & Cymbopogon martini & Flowering plants & India & Essenthya \\
\hline Star anise & Illicium verum & Fruits and seeds & Vietnam & Primavera \\
\hline Tea tree & Melaleuca alternifolia & Leaves and twigs & Australia & Naturando \\
\hline Turmeric & Curcuma longa & Rhizomes & Madagascar & Essenthya \\
\hline Chinese yin yang & $\begin{array}{l}\text { Mix (Eucalyptus globulus, } \\
\text { Cymbopogon citratus, } \\
\text { Caryophylli aetheroleum, Mentha } \\
\text { piperita, Pinus sylvestris, Salvia } \\
\text { rosmarinus, Lavandula officinalis, } \\
\text { Foeniculum vulgare, Salvia } \\
\text { officinalis, Illicium verum, } \\
\text { Mentha arvensis, Abies siberica) }\end{array}$ & - & Austria & Best of Nature \\
\hline Japanese yin yang & Mentha arvensis & Whole plants & Austria & Best of Nature \\
\hline Ylang ylang & Cananga odorata & Flowers & Madagascar & Essenthya \\
\hline
\end{tabular}


EOs (clove, eucalyptus, fennel, lavender, oregano, palmarosa, star anise, tea tree, turmeric, Chinese yin yang, Japanese yin yang, and ylang ylang) were mostly obtained by steam distillation. Instead, bitter orange was extracted by means of cold pressing.

Moreover, pepper EO from Piper niger was obtained by hydrodistillation in Clevenger-type apparatus according to the European Pharmacopeia method 2005.281258 [58]. Briefly, $0.25 \mathrm{~kg}$ of pepper leaves were placed in a spherical bottom flask with a volume of $1 \mathrm{~L}$. For optimization of the process, the volume of EO recovered by the Clevenger system was monitored at regulated intervals until the maximum yield was obtained.

\subsection{GC-MS Analysis}

Diluted EO samples (1:100 $v / v$ in heptane) were analyzed by gas-chromatography-mass spectrometry (GC-MS) using the Trace 1300 GC coupled to the TSQ DUO triple quadrupole mass spectrometer (Thermo Scientific, Walthan, MA, USA) equipped with an electron impact ion source. Samples were injected without derivatization into the DB-5 column, 30-m length, 0.25-mm internal diameter, 0.25- $\mu \mathrm{m}$ film (Thermo Scientific, Walthan, MA, USA) using the 1:10 split mode. The following parameters were used: ionization energy of $70 \mathrm{eV}$, mass range between 50 and $550 \mathrm{~m} / \mathrm{z}$ and interface temperature of $250{ }^{\circ} \mathrm{C}$. The GC oven temperature was as follows: initial oven temperature of $70{ }^{\circ} \mathrm{C}$ and an isotherm for $1 \mathrm{~min}$; subsequently, at $24^{\circ} \mathrm{C} / \mathrm{min}$ to $180^{\circ} \mathrm{C}$ and an isotherm for $2 \mathrm{~min}$, and then reached $280{ }^{\circ} \mathrm{C}$ at $50{ }^{\circ} \mathrm{C} / \mathrm{min}$ where the isotherm was kept for a further $2 \mathrm{~min}$. The carrier gas was helium (He, purity $99.999 \%$ ) with a constant flow of $1.2 \mathrm{~mL} / \mathrm{min}$.

The acquisition data and the control of the instrument were performed through the Chromeleon Chromatography Data System software, CDS (Thermo Scientific, Walthan, MA, USA). The identification of the GC peaks corresponding to the components of the EOs was based on a direct comparison of the retention times and mass spectral data with those of standard compounds, computer matching with the National Institute of Standards and Technology (NIST) library.

Results were presented as a relative percentage of normalized peak area abundances, without the use of correction factors. The percentage data shown were mean values of two injections.

\subsection{Antiyeast Activity on Saccharomyces cerevisiae}

Saccharomyces cerevisiae obtained from the strain collection of the Institute of Research on Terrestrial Ecosystems (IRET) of the National Research Council (CNR) was used to evaluate the antiyeast activity of EOs. Stock culture was maintained at $4{ }^{\circ} \mathrm{C}$ on Malt Extract Agar (malt extract $30 \mathrm{~g} / \mathrm{L}$; peptone $5 \mathrm{~g} / \mathrm{L}$; agar $15 \mathrm{~g} / \mathrm{L}$ ). Inocula were obtained from overnight cultures on MEA plates at $28^{\circ} \mathrm{C}$. S. cerevisiae was grown in malt extract (ME $30 \mathrm{~g} / \mathrm{L}$; peptone $5 \mathrm{~g} / \mathrm{L}$ ) for $24 \mathrm{~h}$ in an orbital shaking incubator at $120 \mathrm{rpm}$ at $28{ }^{\circ} \mathrm{C}$ [59]. The growth was monitored both by measuring the absorbance at $600 \mathrm{~nm}$ and by counting on plates $(\mathrm{CFU} / \mathrm{mL})$.

\subsubsection{Solid Medium Diffusion Method}

A solid medium diffusion procedure using wells in dishes was used to determine the antiyeast activity of all EOs [22,44]. For this, $1 \mathrm{~mL}$ of $S$. cerevisiae suspension with a concentration of $10^{6} \mathrm{CFU} / \mathrm{mL}$ was uniformly spread on a sterile MEA petri dish (diameter $9 \mathrm{~cm}$ ). After inoculum absorption by agar, wells were made using sterile glass tubes (diameter $6 \mathrm{~mm}$ ) which were filled with $10 \mu \mathrm{L}$ of each EO. The disc radius was not included. Negative controls were prepared with only $10 \mu \mathrm{L}$ of DMSO. Petri dishes were incubated at $28^{\circ} \mathrm{C}$ for $48 \mathrm{~h}$; antiyeast activity was evaluated by measuring the diameter of the growth inhibition halos and was expressed in millimeters. All determinations were carried out in triplicate.

\subsubsection{Minimum Inhibitory Concentration}

EOs showing growth inhibition, as clear white zone with diameters equal to or greater than $10 \mathrm{~mm}$ with the solid medium diffusion method, were considered to determine the minimum inhibitory 
concentration (MIC) using a liquid medium. The MIC was defined as the lowest concentration of an EO that resulted in a reduction of $>90 \%$ in the measured absorbance $[15,22,44,60]$.

The microplate bioassay was used to study the antiyeast activity of EOs. The 24-well plates were prepared by dispensing into each well $1.8 \mathrm{~mL}$ of Malt Extract broth and $0.2 \mathrm{~mL}$ of yeast inoculum with a final concentration of $10^{6} \mathrm{CFU} / \mathrm{mL}$. An aliquot $(20 \mu \mathrm{L})$ of each $\mathrm{EO}$ (with concentrations ranging from 0.1 to $1000 \mu \mathrm{L} / \mathrm{mL}$ ) was transferred into a well. Negative controls were prepared adding only $20 \mu \mathrm{L}$ of DMSO to ME medium. The microplates were sealed and incubated on a plate shaker (100 rpm) at $28^{\circ} \mathrm{C}$ for $48 \mathrm{~h}$. The yeast growth was evaluated by measuring the absorbance at $600 \mathrm{~nm}$.

\subsection{Antioxidant Activity}

The radical scavenging activity (RSA) of EOs was evaluated by 2,2'-diphenyl-1-picrylhydrazyl (DPPH) assay according to the procedure of Blois [61]. Briefly, $150 \mu \mathrm{L}$ of each EOs (with a concentration ranging from 0.1 to $1000 \mu \mathrm{L} / \mathrm{mL}$ ) were mixed with $1.35 \mathrm{~mL}$ of $60-\mu \mathrm{M}$ DPPH methanolic solution. The absorbance reduction at $517 \mathrm{~nm}$ of the DPPH was determined continuously for $60 \mathrm{~min}$. The RSA was calculated as a percentage of DPPH discoloration, using the following equation:

$$
\% \text { RSA }=\frac{\left(\mathrm{A}_{\mathrm{DPPH}}-\mathrm{A}_{\mathrm{s}}\right)}{\mathrm{A}_{\mathrm{DPPH}}} \times 100
$$

where $A_{S}$ is the absorbance of the solution when the EO was added and $A_{D P P H}$ is the absorbance of the DPPH solution, as reported [62]. The extract concentration (EC) necessary to achieve $50 \%$ radical DPPH inhibition $\left(\mathrm{EC}_{50}\right)$ was obtained by plotting the RSA percentage as a function of EOs' concentrations and was expressed as microliters per milliliter $(\mu \mathrm{L} / \mathrm{mL})$.

Moreover, in order to classify the EOs, the antioxidant activity index (AAI) was determined as follows:

$$
\mathrm{AAI}=\mathrm{DPPH} \text { concentration in reaction mixture } / \mathrm{EC}_{50}
$$

EOs were categorized as showing poor antioxidant activity (AAI $<0.5)$, moderate $(0.5<\mathrm{AAI}<1.0)$, strong $(1.0<\mathrm{AAI}<2.0)$ and very strong $(\mathrm{AAI}>2.0)$, as reported by Scherer and Godoy [51] and Cautela et al. [52].

\subsection{Statistical Analysis}

Samples were analyzed in triplicate and all results were expressed as mean \pm standard deviation (SD). Means, SD, calibration curves and linear regression analyses $\left(\mathrm{R}^{2}\right)$ were determined using Microsoft Excel 2013 (Microsoft Corporation, Redmond, WA, USA). The multivariate analysis was performed applying Principal Component Analysis (PCA) through XLSTAT Statistical Software using Microsoft Excel 2013.

\section{Conclusions}

From analyzing the chemical composition of the $14 \mathrm{EOs}$, bitter orange was found to consist mainly of monoterpenes while tea tree, eucalyptus, lavender, palmarosa, Chinese yin yang, and Japanese yin yang were characterized by high amounts of monoterpenoids and oxygenated monoterpenes. Sesquiterpenes, on the other hand, were abundant in ylang ylang, while star anise, fennel, clove, and oregano were rich in phenylpropanoids. Turmeric and pepper EOs exhibited a more heterogeneous chemoprofile and could not be associated with a prevalent terpenic class.

Overall, the 14 EOs studied were classified into six different chemotypes according to their chemical compositions and their relative abundances. The exploratory PCA technique allowed us to visualize, by reducing the dimension of the original data, and provide phytochemical relationships among all the EOs studied. Summarizing our results, amongst the 14 EOs studied, clove showed the highest antioxidant activity with an $\mathrm{EC}_{50}$ of $0.36 \mu \mathrm{L} / \mathrm{mL}$, followed by Chinese yin yang, oregano and ylang ylang. Moreover, oregano had the greatest antiyeast properties, inhibiting the growth of 
S. cerevisiae with an MIC of $10 \mu \mathrm{L} / \mathrm{L}$, followed by clove, palmarosa, bitter orange, eucalyptus and Japanese yin yang EOs. Therefore, considering the EOs studied here, clove for its high antioxidant activity and oregano for its great antiyeast activity may have potential uses in the food and beverage sectors to increase shelf life and avoid deterioration.

Author Contributions: Conceptualization, B.L. and F.M.V.; investigation, R.C., D.C., I.F., G.P., and F.M.V.; data analysis, D.C., B.L., G.P., and F.M.V; writing-review and editing, B.L., G.P. and F.M.V. All authors have read and agreed to the published version of the manuscript.

Funding: This research received no external funding.

Acknowledgments: The authors gratefully thank Alessandro di Matteo for carrying out some experiments related to his master's thesis.

Conflicts of Interest: The authors declare no conflict of interest.

\section{References}

1. Raut, J.S.; Karuppayil, S.M. A status review on the medicinal properties of essential oils. Ind. Crop. Prod. 2014, 62, 250-264. [CrossRef]

2. Prakash, B.; Kedia, A.; Mishra, P.K.; Dubey, N.K. Plant essential oils as food preservatives to control moulds, mycotoxin contamination and oxidative deterioration of agri-food commodities-Potentials and challenges. Food Control 2015, 47, 381-391. [CrossRef]

3. Bakkali, F.; Averbeck, S.; Averbeck, D.; Idaomar, M. Biological effects of essential oils-a review. Food Chem. Toxicol. 2008, 46, 446-475. [CrossRef] [PubMed]

4. Al-Tamimi, A.M.; Rastall, B.; Abu-Reidah, M.I. Chemical composition, cytotoxic, apoptotic and antioxidant activities of main commercial essential oils in Palestine: A comparative study. Medicines 2016, 3, 27. [CrossRef]

5. Bassolé, I.H.N.; Juliani, H.R. Essential oils in combination and their antimicrobial properties. Molecules 2012, 17, 3989-4006. [CrossRef]

6. Hyldgaard, M.; Mygind, T.; Meyer, R.L. Essential oils in food preservation: Mode of action, synergies, and interactions with food matrix components. Front. Microbiol. 2012, 3, 12. [CrossRef]

7. Dhifi, W.; Bellili, S.; Jazi, S.; Bahloul, N.; Mnif, W. Essential oils' chemical characterization and investigation of some biological activities: A critical review. Medicines 2016, 3, 25. [CrossRef] [PubMed]

8. Guenther, E. The Essential Oils; D. Van Nostrand: New York, NY, USA, 1948.

9. Pandey, A.K.; Kumar, P.; Singh, P.; Tripathi, N.N.; Bajpai, V.K. Essential oils: Sources of antimicrobials and food preservatives. Front. Microbiol. 2017, 7, 2161. [CrossRef]

10. Burt, S. Essential oils: Their antibacterial properties and potential applications in foods-A review. Int. J. Food Microbiol. 2004, 94, 223-253. [CrossRef]

11. Dima, C.; Dima, S. Essential oils in foods: Extraction, stabilization, and toxicity. Curr. Opin. Food Sci. 2015, 5, 29-35. [CrossRef]

12. Atares, L.; Chiralt, A. Essential oils as additives in biodegradable films and coatings for active food packaging. Trends Food Sci. Technol. 2016, 48, 51-62.

13. Hernández, A.; Pérez-Nevado, F.; Ruiz-Moyano, S.; Serradilla, M.J.; Villalobos, M.C.; Martín, A.; Córdoba, M.G. Spoilage yeasts: What are the sources of contamination of foods and beverages? Int. J. Food Microbiol. 2018, 286, 98-110.

14. Hutzler, M.; Riedl, R.; Koob, J.; Jacob, F. Fermentation and spoilage yeasts and their relevance for the beverage industry-A review. Brew. Sci. 2012, 65, 33-52.

15. Tserennadmid, R.; Takó, M.; Galgóczy, L.; Papp, T.; Pesti, M.; Vágvölgyi, C.; Almassy, K.; Krisch, J. Anti-yeast activities of some essential oils in growth medium, fruit juices and milk. Int. J. Food Microbiol. 2011, 144, 480-486. [PubMed]

16. Fleet, G.H. Yeast spoilage of foods and beverages. In The Yeasts Handbook; Kurtzman, C.P., Fell, J.W., Boekhout, T., Eds.; Elsevier: Amsterdam, The Netherlands, 2011; Volume 1, pp. 53-63. Available online: https://www.sciencedirect.com/science/article/pii/B9780444521491000057 (accessed on 6 May 2020).

17. Nyman, P.J.; Diachenko, G.W.; Perfetti, G.A.; McNeal, T.P.; Hiatt, M.H.; Morehouse, K.M. Survey results of benzene in soft drinks and other beverages by headspace gas chromatography/mass spectrometry. J. Agric. Food Chem. 2008, 56, 571-576. 
18. Singh, G.; Singh, O.P.; Rao, G.P.; Sharma, S.R. The vapour action of essential oils and monoterpenoids against pathogenic fungi. Sugar Tech. 2002, 4, 69-71.

19. Ultee, A.; Bennik, M.H.J.; Moezelaar, R. The phenolic hydroxyl group of carvacrol is essential for action against the food-borne pathogen Bacillus cereus. Appl. Environ. Microbiol. 2002, 68, 1561-1568.

20. Gill, A.O.; Holley, R.A. Mechanisms of bactericidal action of cinnamaldehyde against Listeria monocytogenes and of eugenol against L. monocytogenes and Lactobacillus sakei. Appl. Environ. Microbiol. 2004, 70, 5750-5755. [PubMed]

21. Baratta, M.T.; Dorman, H.D.; Deans, S.G.; Figueiredo, A.C.; Barroso, J.G.; Ruberto, G. Antimicrobial and antioxidant properties of some commercial essential oils. Flavour. Frag. J. 1998, 13, 235-244.

22. Teixeira, B.; Marques, A.; Ramos, C.; Neng, N.R.; Nogueira, J.M.; Saraiva, J.A.; Nunes, M.L. Chemical composition and antibacterial and antioxidant properties of commercial essential oils. Ind. Crop. Prod. 2013, 43, 587-595.

23. Sacchetti, G.; Maietti, S.; Muzzoli, M.; Scaglianti, M.; Manfredini, S.; Radice, M.; Bruni, R. Comparative evaluation of 11 essential oils of different origin as functional antioxidants, antiradicals and antimicrobials in foods. Food Chem. 2005, 91, 621-632. [CrossRef]

24. Misharina, T.; Samusenko, A. Antioxidant properties of essential oils from lemon, grapefruit, coriander, clove, and their mixtures. Appl. Biochem. Microbiol. 2009, 44, 642-647. [CrossRef]

25. Noumi, E.; Snoussi, M.; Hajlaoui, H.; Trabelsi, N.; Ksouri, R.; Valentin, E.; Bakhrouf, A. Chemical composition, antioxidant and antifungal potential of Melaleuca alternifolia (tea tree) and Eucalyptus globulus essential oils against oral Candida species. J. Med. Plant. Res. 2011, 5, 4147-4156.

26. Zhang, X.; Guo, Y.; Guo, L.; Jiang, H.; Ji, Q. In vitro evaluation of antioxidant and antimicrobial activities of Melaleuca alternifolia essential oil. Biomed. Res. Int. 2018, 2018, 1-8. [CrossRef]

27. Freire, J.M.; Cardoso, M.G.; Batista, L.R.; Andrade, M.A. Essential oil of Origanum majorana L., Illicium verum Hook. f. and Cinnamomum zeylanicum Blume: Chemical and antimicrobial characterization. Rev. Bras. Plantas Med. 2011, 13, 209-214. [CrossRef]

28. Cautela, D.; Vella, F.M.; Castaldo, D.; Laratta, B. Characterization of essential oil recovered from fennel horticultural wastes. Nat. Prod. Res. 2019, 33, 1964-1968. [CrossRef]

29. Bourgou, S.; Rahali, F.Z.; Ourghemmi, I.; Saïdani Tounsi, M. Changes of peel essential oil composition of four Tunisian citrus during fruit maturation. Sci. World J. 2012, 2012, 1-10. [CrossRef] [PubMed]

30. Azhdarzadeh, F.; Hojjati, M. Chemical composition and antimicrobial activity of leaf, ripe and unripe peel of bitter orange (Citrus aurantium) essential oils. Food Sci. Nutr. 2016, 3, 43-50. [CrossRef]

31. Dorman, H.D.; Surai, P.; Deans, S.G. In vitro antioxidant activity of a number of plant essential oils and phytoconstituents. J. Essent. Oil Res. 2000, 12, 241-248. [CrossRef]

32. Menon, A.N.; Padmakumari, K.P. Essential oil composition of four major cultivars of black pepper (Piper nigrum L.) - IV. J. Essent. Oil Res. 2005, 17, 206-208.

33. Tomaino, A.; Cimino, F.; Zimbalatti, V.; Venuti, V.; Sulfaro, V.; De Pasquale, A.; Saija, A. Influence of heating on antioxidant activity and the chemical composition of some spice essential oils. Food Chem. 2005, 89, 549-554. [CrossRef]

34. Jirovetz, L.; Buchbauer, G.; Stoilova, I.; Stoyanova, A.; Krastanov, A.; Schmidt, E. Chemical composition and antioxidant properties of clove leaf essential oil. J. Agric. Food Chem. 2006, 54, 6303-6307. [CrossRef]

35. Andrade, M.T.B.F.; Conti, B.J.; Santiago, K.B.; Fernandes, A.; Sforcin, J.M. Cymbopogon martinii essential oil and geraniol at non-cytotoxic concentrations exerted immunomodulatory/anti-inflammatory effects in human monocytes. J. Pharm. Pharmacol. 2014, 66, 1491-1496.

36. Pandey, A.K.; Rai, M.K.; Acharya, D. Chemical composition and antimycotic activity of the essential oils of corn mint (Mentha arvensis) and lemon grass (Cymbopogon flexuosus) against human pathogenic fungi. Pharm. Biol. 2003, 41, 421-425. [CrossRef]

37. Hussain, A.I.; Anwar, F.; Nigam, P.S.; Ashraf, M.; Gilani, A.H. Seasonal variation in content, chemical composition and antimicrobial and cytotoxic activities of essential oils from four Mentha species. J. Sci. Food Agric. 2010, 90, 1827-1836. [CrossRef]

38. Cavanagh, H.M.A.; Wilkinson, J.M. Biological activities of lavender essential oil. Phytother. Res. 2002, 16, 301-308. [CrossRef] 
39. Maietti, S.; Rossi, D.; Guerrini, A.; Useli, C.; Romagnoli, C.; Poli, F.; Bruni, R.; Sacchetti, G. A multivariate analysis approach to the study of chemical and functional properties of chemo-diverse plant derivatives: Lavender essential oils. Flavour Frag. J. 2013, 28, 144-154. [CrossRef]

40. Sienkiewicz, M.; Glowacka, A.; Kowalczyk, E.; Wiktorowska-Owczarek, A.; Jozwiak-Bębenista, M.; Lysakowska, M. The biological activities of cinnamon, geranium and lavender essential oils. Molecules 2014, 19, 20929-20940. [CrossRef] [PubMed]

41. Granato, D.; Putnik, P.; Kovačević, D.B.; Santos, J.S.; Calado, V.; Rocha, R.S.; Gomes Da Cruz, A.; Jarvis, B.; Rodionova, O.Y.; Pomerantsev, A. Trends in chemometrics: Food authentication, microbiology, and effects of processing. Compr. Rev. Food Sci. Food Saf. 2018, 17, 663-677. [CrossRef]

42. Yu, Y.; Bai, J.; Chen, C.; Plotto, A.; Baldwin, E.A.; Gmitter, F.G. Comparative analysis of juice volatiles in selected mandarins, mandarin relatives and other citrus genotypes. J. Sci. Food Agric. 2018, 98, 1124-1131. [CrossRef]

43. Kharbach, M.; Marmouzi, I.; El Jemli, M.; Bouklouze, A.; Vander Heyden, Y. Recent advances in untargeted and targeted approaches applied in herbal-extracts and essential oils fingerprinting-A review. J. Pharm. Biomed. Anal. 2020, 177, 112849. [CrossRef]

44. Souza, E.L.; Stamford, T.L.M.; Lima, E.O.; Trajano, V.N. Effectiveness of Origanum vulgare L. essential oil to inhibit the growth of food spoiling yeasts. Food Control 2007, 18, 409-413. [CrossRef]

45. Di Pasqua, R.; Hoskins, N.; Betts, G.; Mauriello, G. Changes in membrane fatty acids composition of microbial cells induced by addiction of thymol, carvacrol, limonene, cinnamaldehyde, and eugenol in the growing media. J. Agric. Food Chem. 2006, 54, 2745-2749. [CrossRef]

46. Bennis, S.; Chami, F.; Chami, N.; Bouchikhi, T.; Remmal, A. Surface alteration of Saccharomyces cerevisiae induced by thymol and eugenol. Lett. Appl. Microbiol. 2004, 38, 454-458. [CrossRef] [PubMed]

47. Chen, W.; Viljoen, A.M. Geraniol-A review of a commercially important fragrance material. S. Afr. J. Bot. 2010, 76, 643-651. [CrossRef]

48. Sharma, Y.; Khan, L.A.; Manzoor, N. Anti-candida activity of geraniol involves disruption of cell membrane integrity and function. J. Mycol. Med. 2016, 26, 244-254. [CrossRef]

49. dos Santos, A.S.; Santos Silva, G.; Souza Silva, K.V.; de Oliveira Lima, M.I.; Mendes Arrua, J.M.; de Oliveira Lima, E.; de Oliveira Pereira, F. Antifungal activity of geraniol and citronellol against food-relevant dematiaceous fungi Cladosporium spp. Rev. Inst. Adolfo Lutz 2017, 76, e1732.

50. Bassolé, I.H.N.; Lamien-Meda, A.; Bayala, B.; Tirogo, S.; Franz, C.; Novak, J.; Nebié, R.C.; Dicko, M.H. Composition and antimicrobial activities of Lippia multiflora Moldenke, Mentha x piperita L. and Ocimum basilicum L. essential oils and their major monoterpene alcohols alone and in combination. Molecules 2010, 15, 7825-7839. [CrossRef]

51. Scherer, R.; Godoy, H.T. Antioxidant activity index (AAI) by the 2,2-diphenyl-1-picrylhydrazyl method. Food Chem. 2009, 112, 654-658. [CrossRef]

52. Cautela, D.; Vella, F.M.; Laratta, B. The effect of processing methods on phytochemical composition in bergamot juice. Foods 2019, 8, 474. [CrossRef]

53. Wei, A.; Shibamoto, T. Antioxidant/lipoxygenase inhibitory activities and chemical compositions of selected essential oils. J. Agric. Food Chem. 2010, 58, 7218-7225. [CrossRef]

54. Ruberto, G.; Baratta, M.T. Antioxidant activity of selected essential oil components in two lipid model systems. Food Chem. 2000, 69, 167-174. [CrossRef]

55. Liu, R.H. Health benefits of fruit and vegetables are from additive and synergistic combinations of phytochemicals. Am. J. Clin. Nutr. 2003, 78, 517S-520S. [CrossRef]

56. Bilotto, S.; Spagnuolo, C.; Russo, M.; Tedesco, I.; Laratta, B.; Russo, G.L. Dietary phytochemicals in chemoprevention of cancer: An update. Immunol. Endocr. Metab. Agents Med. Chem. 2013, 13, $2-24$. [CrossRef]

57. Dahham, S.S.; Tabana, Y.M.; Iqbal, M.A.; Ahamed, M.B.; Ezzat, M.O.; Majid, A.S.; Majid, A.M. The anticancer, antioxidant and antimicrobial properties of the sesquiterpene $\beta$-caryophyllene from the essential oil of Aquilaria crassna. Molecules 2015, 20, 11808-11829. [CrossRef] [PubMed]

58. European Directorate for the Quality of Medicines and Health Care. European 121 Pharmacopeia Method 2.08.12: Essential Oils in Herbal Drugs; Council of Europe: Strasbourg, France, 2015.

59. Vella, F.M.; Laratta, B. UV-based evaluation of ergosterol for monitoring the fungal exposure in Italian buffalo farms. FEMS Microbiol. Lett. 2017, 364, fnx224. [CrossRef] 
60. Hili, P.; Evans, C.S.; Veness, R.G. Antimicrobial action of essential oils: The effect of dimethylsulphoxide on the activity of cinnamon oil. Lett. Appl. Microbiol. 1997, 24, 269-275. [CrossRef]

61. Blois, M.S. Antioxidant determinations by the use of a stable free radical. Nature 1958, 181, 1199-1200. [CrossRef]

62. Vella, F.M.; De Masi, L.; Calandrelli, R.; Morana, A.; Laratta, B. Valorization of the agro-forestry wastes from Italian chestnut cultivars for the recovery of bioactive compounds. Eur. Food Res. Technol. 2019, 245, 2679-2686. [CrossRef]

Sample Availability: Voucher specimens are available at IRET-CNR by the authors.

Publisher's Note: MDPI stays neutral with regard to jurisdictional claims in published maps and institutional affiliations.

(C) 2020 by the authors. Licensee MDPI, Basel, Switzerland. This article is an open access article distributed under the terms and conditions of the Creative Commons Attribution (CC BY) license (http://creativecommons.org/licenses/by/4.0/). 